\title{
INTEGRATION OF ECONOMIC APPRAISAL AND HEALTH CARE POLICY IN A HEALTH INSURANCE SYSTEM; THE DUTCH CASE*
}

\author{
FRANS RUTtEN $^{1}$ and JAN-WILLEM VAN DER LINDEN ${ }^{2}$ \\ ${ }^{1}$ Institute for Medical Technology Assessment, Erasmus University Rotterdam, P.O. Box 1738, 3000 DR \\ Rotterdam, The Netherlands and ${ }^{2}$ Health Insurance Executive Board, P.O. Box 396, 1180 BD Amstelveen, \\ The Netherlands
}

\begin{abstract}
This article discusses the role of economic appraisal in insurance based health care systems, taking the case of the Netherlands as an example. The public health insurance system in this country is governed by the Health Insurance Executive Board, which policies are firmly based on the results of economic appraisal. Furthermore, reimbursement policies regarding pharmaceutical products are described, emphasizing again the position of information from economic appraisal in these policies. The article concludes with the identification of some trends in health policy and in the way it is supported by economic appraisal studies.
\end{abstract}

Key words-economic appraisal, health policy, drug reimbursement

\section{INTRODUCTION}

Countries exhibit a considerable diversity of health care institutions and it is therefore not instructive to conduct a general discussion on the role of economic appraisal in health care policy. Indeed policies to guide the diffusion of health care technologies are quite different across health care systems and involve quite different actors. Hurst [1] suggests that health care systems can be described as comprising seven different mixtures of major subsystems of finance and delivery of health care. One distinction is related to the finance side and consists of three categories: out of pocket payment for health care, voluntary or private health insurance usually with choice of insurer and compulsory or public health insurance, usually without effective choice of insurer. The latter two categories may be combined with one of three methods of paying providers: indirect payment of providers through reimbursement of patients, direct payment of providers by contracts and direct payment of providers by global budget and salaries in a vertically integrated system. These characteristics in finance and delivery determine the degree and way of government regulation in these health care systems, either centralized or decentralized, and, as often in public insurance systems, with a delegation of power to quasi-governmental institutions.

Depending on the character of the system and the actors involved in health policy the role of economic

*Paper prepared for an EC Workshop on From Results to Action: the Role of Economic Appraisal in Developing Policy for Health Technology, Heraklion, Crete, 8-10 October 1992 evaluation will be different. Its role will be more clear when firm regulatory mechanisms are in place than in the case of a decentralized system where contracts between local financers and local providers determine the degree of diffusion of medical technologies. The specifics of resource allocation in a particular health care system may even determine the type of economic analysis needed. The perspective from which a study is undertaken differs according to whether central government or a local health care insurer is the principal user of the results, and in a competitive environment one tends to rely on cost-benefit analysis (willingness to pay determining the value of outcomc), whilc in a socialized system cost-effectiveness or cost-utility analysis is more relevant. In Section 2 we will discuss the options for control in a compulsory health insurance system as is the dominant mode in central Europe.

In Sections 35 we will consider the Dutch system, which key feature is also compulsory social health insurance. Under this system the whole population is insured for chronic care and about $60 \%$ of the population is compulsorily insured for acute care. The remainder of the population relies on voluntary insurance for acute care. Under the public insurance systems the GP's are paid by capitation, specialists mainly by fee for service and hospitals by per diem payments under a global budget, which is determined through a budget formula and based on negotiations with local financers regarding variable costs. Private insurance reimburses patients who are billed on the basis of fee for service. Price and quantity of health care services financed through either insurance system are closely regulated by quasi-governmental 
institutions. One of these is the Health Insurance Executive Board (HIEB), which is responsible for governing and controlling the public insurance system. From the mid eighties onward the Board's policy has been to use the results of economic evaluation in a variety of ways, which will be discussed in Section 3.

In Section 4 special attention will be given to the role of economic evaluation in promoting rational utilization of medicines. In contrast to the situation in other countries (compare the guidelines in Australia [2] and Ontario) there is not yet an official policy positioning economic evaluation in this area. We will give a number of examples, however, where the results of such studies have been quite influential. In Section 5 we will briefly touch on future developments within the Dutch health care system and their likely consequences for the role of economic evaluation in health policy.

Finally, in Section 6 we will identify a number of common problems related with the use of results of economic evaluation in health policy and formulate some lessons to be learned.

\section{ECONOMIC APPRAISAL AND HEALTH POLICY}

Economic evaluation may concern a technology in the early phase of its diffusion or one which diffusion has already progressed. In the former case regulation can be of a more directive nature, but the economic appraisal may be limited by lack of data. In the latter case more reliable data will be available, but it may be more difficult to influence the use of an established technology. Table 1 states the options for control of the diffusion of medical technologies, which may be supported by the results of an economic appraisal. Note that these are all regulatory mechanisms, which should be enforced during the early phase of the life cycle of a new technology. To improve the rational use of existing technologies incentives should be incorporated in the system, such as prospective reimbursement of hospitals forcing management to install internal budgeting procedures and incentives towards efficiency for budget holders.

Table 1 suggests that economic appraisal may support the operation of all regulatory mechanisms mentioned with the exception of pre-market controls for drugs and devices. Efficacy and safety are the main criteria for deciding on admittance of a technology into the market and there are no compelling arguments to add any other criteria. In many countries decisions on instituting national screening programmes are supported by evidence from economic studics, and indecd more so than those on curative programmes. For instance, more than $90 \%$ of the recent Medline-literature on 'costs' and 'cancer' refers to screening topics. Reimbursement decisions on new curative interventions are only occasionally supported by economic analysis, while in many countries economic studies have influenced reimbursement decisions concerning medicines. Economic appraisal may play a role in pricing of medicines, but this depends on the general pricing policies in a health care system and relates to the way in which reimbursement decisions are based on economic arguments. For instance, if no pricing policy exists, rational use of medicines can still be promoted through the use of economic indicators, which are themselves dependent on price, in reimbursement decisions. And finally, licensing policies concerning for instance advanced equipment like MRI may benefit from economic analysis, especially when studies on economies of scale are incorporated in such analysis.

As we will see below these regulatory mechanisms are all used and supported by economic appraisal in Dutch health care policy, with the notable exception of pricing of medicines. Most prominent in this respect are policies as developed within the Health Insurance Executive Board, and these will be discussed in the next section.

\section{THE GENERAL POLICY OF THE HEALTH INSURANCE EXECUTIVE BOARD}

To explain the HIEB policy it is important to note that compulsory health insurance in the Netherlands is regulated by two laws: the Compulsory Health Insurance Act and the General Act on Extraordinary Medical Expenses, the first covering about $60 \%$ of the population and the latter the whole population. The criterion used to judge whether a particular new intervention should be added to the existing benefit packages under these laws has always been whether such new intervention was generally accepted by the medical profession. Confronted with an increasing gap between financial resources and opportunities from advancing medical technology, the HIEB developed a two-stage strategy in the mid eighties to make a halt to the systematic extension of the benefit

Table 1. Options for control of the diffusion of medical technologies

\begin{tabular}{lc}
\hline Regulatory mechanism & Role of economic appraisal \\
\hline Pre-market controls for drugs and devices & \\
Reimbursement from public insurance funds & ++ \\
-preventive programs & + \\
- new curative interventions & ++ \\
-medicines (NCE's) & + \\
Pricing of medicines & + \\
\hline
\end{tabular}


packages and to rationalize policy making in this matter.

First, the HIEB decided to designate certain technologies as emerging and to control their diffusion. For these technologies a three-step procedure was adopted: diffusion of the new technology should be limited and controlled, a medical technology assessment should be carried out simultaneously, and finally, a decision about inclusion of the technology in the benefit packages should be made on the basis of the results of such medical technology assessment. This procedure was followed for heart transplant, liver transplant, breast cancer screening and in vitro fertilization. Based on the results of those studies the HIEB could give well documented advice to include heart transplants in the benefit package, to postpone such a decision in case of the liver transplant, to introduce breast cancer screening for women older than 50 years of age and to postpone the final decision on in vitro fertilization until there is more clarity as to the place of in vitro fertilization in the benefit package of the new insurance system [3].

Although this policy had been successful, it was based on the assumption that central decision making concerning the flow of money could decisively interfere in the process of the introduction of a new medical technology. In some cases it turned out that this was difficult, especially when the co-operation of the clinicians was lacking. For instance, the introduction of the heart transplant programme was somewhat rapid as one university hospital just started doing their own heart transplants without formal approval of the government. Secondly, one became aware that clinicians should be made familiar with the idea that evaluation is a natural step before introduction at a wider scale and that participating in such evaluation sometimes provide the only opportunity to provide a new intervention or programme (as was the case with the technologies mentioned above). And finally, not only the question which technique to in- or exclude from the benefit package should be posed, but also for which indications a particular technology would be cost-effective?

Therefore, the HIEB choose to initiate yet another strategy directed to a more thorough investigation of the possibilities to increase cost-effective and appropriate use of new and existing forms of medical care. This strategy focuses more on the cost-effective application of technologies and procedures and should therefore be targeted especially to the clinicians, who are responsible for resource allocation at the patient level. This strategy led to a change in both Acts in the sense, that the concept of a generally accepted standard for the medical profession was extended towards a standard, which is to be determined through debate between the medical profession and the financers of health care including the government. Application of such newly defined standard to each case would imply a great need for cost-effectiveness analyses.

In 1988 the HIEB, together with the Ministries of
Health and of Education and Science, started a programme of 'investigative medicine'. Investigative medicine is a clinical evaluation programme concerning new and established medical technologies, providing more opportunity for supporting policy decisions. Such policy decision could concern:

- acceptation of a new medical technology or procedure or withdrawing an existing one as a benefit in kind in the Compulsory Health Insurance Act; restriction of the indication to qualify for a benefit in kind.

- improving the efficiency or effectiveness of the use of a medical procedure by means of legislation or through-agreement on a 'protocol';

- a planning decision in connection with the Hospital Provisions' Act.

Each year 36 million Dutch guilders are available to allocate to projects in this programme and researchers/clinicians may each year apply for funds by submitting a proposal which should follow the following general criteria:

- the results of the projects should contribute to decision-making concerning a particular health care intervention;

- a well-designed study plan to compare such intervention with the most cost-effective alternative;

- a thorough evaluation of both effectiveness and costs and, if appropriate, also judicial, organizational and ethical aspects;

- a three-years research period.

Excluded from the 'investigative medicine' initiative are fundamental research, improvement of treatment without evaluation and clinical trials with new pharmaceuticals and new equipment. For new medical technologies and procedures efficacy should be proven; for established technologies the question of effectiveness could be part of the evaluation.

After 5 years experience with the programme a number of positive conclusions can be made, as the first studies have been finished last month and others will follow soon. Many ongoing studies have already produced publications. It can be foreseen now that studies in this programme will contribute to the decisions of the government and to professional agreement in formulating treatment protocols, as is for instance the case with diagnostics of thrombosis which will decrease $66 \%$ of present hospital treatment, which involves many false positives. Another positive consequence is the controlled introduction of for instance pancreas and lung transplantation and ECMO. A third contribution of studies in this programme is in decision making about the indicationarea of growth factors (GM-CSF) and autologous bonemarrow transplantations.

Furthermore, medical technology assessment as an interdisciplinary effort flourishes in the Netherlands, due both to the funding programme itself and to the increasing interest from clinicians and policy makers. This field of investigative medicine has gained in 
importance and in status. On the other hand, there seems to be an overemphasis on high technology as new medical interventions are being evaluated in more than $80 \%$ of the cases, so less work is done on established technologies. Furthermore, $70 \%$ of the proposals concern therapy and $30 \%$ concern diagnostics. The fields of oncology, cardiovascular disease, organ transplantation, neurology and gynaecology/obstetrics dominate. But there is some evidence that dissemination of the result of this programme is relatively good as peer clinicians are involved in these projects from the beginning. In the future, the Committee for Investigative Medicine wants to combine the top-down and bottom-up approach by selecting some topics for evaluation themselves and asking for tenders from research institutes.

\section{THE POLICY REGARDING MEDICINES}

Health policy makers may influence the use of medicines in health care in a variety of ways. In most countries there are barriers to entrance into the market (pre-market controls), there may be restrictions to reimbursement, there may be an active pricing policy, and there may be a variety of indirect ways of influencing the behaviour of the prescribing physician. And there are sometimes other objectives than improving the efficiency of health care programmes, e.g. when countries want to foster investment in plants and research. In discussing these options, the role of economic evaluation and the Dutch experience in this context, it is useful to make a distinction between extramural use of medicines and the use of medicines within the hospital. For both types of medicines the registration procedure is similar. As in most countries the only criteria in the context of registration are 'efficacy' and 'safety'. Economic evaluation is not foreseen to play a role in this phase.

Regarding medicines used in an ambulatory setting in the Netherlands there exists an open system of reimbursement of medicines, meaning that registered drugs are in principle being reimbursed. Sweden and the Netherlands are generally considered as countries with a rather liberal system with respect to prescribing [4]. Increasingly, however, medicines are allocated to a list (Appendix 3, agreement pharmaceutical care in public health insurance), where there are restrictions to the use of medicines in this respect. These restrictions may relate to certain indications, but may also relate to the discipline of the prescribing physician. Also for a number of medicines on this list, permission to use them should be granted from the public insurance agency in advance. This list contains products like interferon, growth factors and erythropoietin (EPO), which are costly as they are produced by DNA recombinant analysis. Examples of drugs in this list are for instance AZT and the new cholesterol lowering agents. In some cases where no permission a priori is needed such as in the case of cholesterol lowering drugs, the HIEB is monitoring sales of these medicines at a global level comparing a priori expectations based on epidemiological analysis and actual sales in the market. If the discrepancy becomes too large, a procedure to require permission in advance for prescribing these cholesterol lowering drugs may be instituted.

Economic evaluation has played and will play a role in this policy of conditional reimbursement. For instance, at the time of deciding about reimbursement of Zocor (the new cholesterol lowering agent of MSD) the HIEB had at its disposal an advice by the Health Council and an economic evaluation carried out at the initiative of the producer of the drug, MSD. The results of this study were taken into account when deciding on the conditions for reimbursement of Zocor. The HIEB is now discussing a change from an 'open system' to a 'closed system', meaning that new innovative drugs (New Chemical Entities) will only be reimbursed after an explicit decision by the Board (followed by approval of the Ministry of Health, Welfare and Cultural Affairs). It is foreseen that economic appraisal will support such decision making process and that guidelines about the format of such appraisal (as in Australia and Ontario) will be issued.

Another issue in reimbursement is the introduction in 1991 of a new reimbursement system for medicines based on the concept of reference prices. For classes of medicines with the same therapeutic effectiveness a reference price will be determined and the insured will have to pay the difference between the actual price and the reference price. This reference price is chosen in such a way that there is enough opportunity for the insured to receive proper medication without any co-payment. One of the assumptions underlying this reference price system is that price increases because of innovation by way of introducing me-too products can be mitigated. Furthermore, the system is believed to make physician prescribers more aware of the prices of medicines. As a consequence of introducing this system the price index of drugs and pharmaceutical products decreased with an initial $3 \%$. In some sectors, however, producers and importers of drugs increased their prices considerably (anti-conceptives) creating a situation of considerable co-payment. Prescribers, pharmacists and insured reacted by changing over to cheaper products, which made the producers and importers, who initially increased their prices, to reduce them again toward or just under the reference price. In general one can observe an increasing market share of generic and parallel imported drugs in 1992. Sofar, there has not been a role for economic appraisal in regard to this reference price system of reimbursing drugs. Some pharmaceutical companies, however, are in the process of assembling economic evidence, trying to demonstrate that their product is different in its cost-effectiveness profile from the other drugs, with which they are placed in the same reference group. 
There is no evidence yet that such arguments may change the position of a particular drug in the reference price system.

By far the most important contribution to increasing the efficiency of drug use lies in the indirect ways of influencing prescribing behaviour of physicians. The role of individual public health insurance agencies is rather limited as they have few opportunities to create incentives for physicians to be efficient (for instance, they can not end a contractual relationship with a physician). One of the items in the plans for a revision of the Dutch health insurance system is to introduce more competition between providers and free contracting of health insurance agencies with those providers. This leaves more opportunity for financers to incorporate incentives for efficiency in contracts with providers.

Another important innovation is the start of pharmacotherapeutic commissions, in which pharmacists and prescribing physicians systematically discuss protocols for prescribing drugs. During an experiment with such commission in a particular region about $87 \%$ of general practitioners participated in the discussions, as well as $96 \%$ of the pharmacists. Reports about cost-effective drug use may be inputs in such discussion. Furthermore, the HIEB publishes every year a pharmacotherapeutic advice concerning the price/quality ratio of medicines to inform the practitioners.

Hospital drugs are normally reimbursed from hospital budgets as is the case with other expenditure within the hospital. However, it is possible to obtain an additional budget for costly drugs or biotech products. Such addition to the budget was given for erythropoietin (EPO) in relation to its use for dialysis patients. In such case there is an earmarked flow of money to the hospital specific for the use of EPO in dialysis patients. In this case economic appraisal only played a minor role, but the HIEB secretariat is assembling information on the impact of EPO on quality of life in order to continuously evaluate their policy in this respect. Because of the high costs of some new biotech products like G-CSF pharmaceutical companies are assembling economic data to facilitate their discussions with the HIEB on the position of their products in hospital care.

From the observations above one may conclude, that there is a trend towards a greater role of economic appraisal in health policy decisions on the reimbursement of drugs and biotech products. The HIEB secretariat is now developing its policies for the nineties and considering a fundamental change towards formalizing the role of economic evaluation in this area. The outcome of the discussion is possibly that only medicines that are innovative and offer a really effective therapy should be reimbursed. In other cases a medicine will be clustered in the reference price system of will be excluded for reimbursements.

\section{DECENTRALIZATION IN HEALTH POLICY}

In 1988 the Dutch government decided to radically reform the financial structure and the organization of the health care system. Two major components of this reform are the introduction of a national health insurance scheme and regulated competition among insurers and among providers [5]. Execution of this plan started in 1989 and was expected to be completed in 1994. The latter will not be achieved since there is political debate on whether and, if so, in what form the plans should continue to be carried out. With respect to health insurance the division between compulsory insurance under the Health Insurance Act, private insurance and the insurance schemes for public servants is planned to disappear. Instead, there will be a national health insurance scheme offering a relatively wide range of benefits. Some first steps towards such insurance system are being set and more flexibility is introduced concerning the way of offering these benefits to the insured population. For instance, in the old system specific types of treatment to be delivered by specific health care providers were narrowly circumscribed in the benefit package, while in the new system insurers are free to substitute one form of care for the other as long as these are serving similar purposes.

This planned health reform can be characterized as a change from a highly government regulated system towards a system where resource allocation is mainly determined through contracting of individual providers and insurers. Central government regulation will become less important and so will the need to support these policies with evidence from economic appraisal. Instead, we will sec a growing necd for this kind of information with the regional and local parties in health care. Indeed, Henshall and Drummond [6] argue that competitive forces may encourage individual parties to consider costeffectiveness information. On balance, however, we predict that it will take some time before the new parties in health care are able to appreciate the benefits of economic appraisal and position the outcomes of such studies in their own policy-making processes. So the level of activities in economic appraisal may fall! First, problems can be expected when dealing with medical technologies, which have a supra-regional characteristic and can only meaningfully be tackled at a national level. Furthermore, individual insurers or health care organizations may find it difficult to finance economic appraisal studies when these yield results of a more general value than only for one particular insurer or health care organization. A free rider problems emerges when such information is also freely available to others, and if this is not the case and the information is clearly marked as having a commercial value, there will be obvious problems with the dissemination of such information. And finally, the danger of relying too much on market forces is that the role of consumer 
preferences will become dominant, and this may conflict with utilitarian values as seen from society's perspective such as cost-effectiveness [7]. Maybe patient and consumer organizations may step in and resume some of the responsibilities that are being left by the government. If so, they may become more important in initiating economic appraisal studies and promoting dissemination of results. In conclusion, there are great uncertainties about the role of economic appraisal in health policy when the reform plans will be actually executed.

\section{OBSERVATIONS AND CONCLUSIONS}

Some observations from the Dutch experience as described above can be made and some tentative conclusions can be drawn:

- Dutch policies concerning the initiation of economic appraisal studies seem to converge towards a mixed top-down bottom-up approach. The former (top-down) has the clear advantage of positioning economic appraisal firmly in health care policy as in the case of the initial HIEB strategy, while the latter (bottom-up) has the advantage of being better integrated in the clinical and research community which may further the dissemination of the results of economic appraisal among clinicians. Furthermore, the HIEB policies and, particularly, the investigative medicine' program have contributed considerably to the development of expertise in economic appraisal and, more generally, in medical technology assessment and to the cooperation between clinicians and evaluation-researchers;

- The role of economic appraisal in deciding on the reimbursement of drugs and biotech products will become more important and a similar development towards formalizing this role as in Australia and Ontario is expected in the coming years. This is not only in line with EC-transparency rules, but would also provide a clear signal to industry to strive towards cost-effective products and to assemble data on cost-effectiveness at an early stage [6]. There is a tendency to reimburse only really innovative medicines which offer new therapeutic opportunities as well as those medicines which have a favourable costeffectiveness profile;

- The timing of a study has shown to be an important issue. Looking back at the initial HIEB initiatives for economic appraisal, the liver transplant study was initiated too early as the technology was still developing, while the IVF study was probably too late to achieve maximum momentum in policy. An early warning function, as is assumed by the Dutch Health Council, would contribute to gaining time for selecting the right topics and determining the timing for carrying out appraisals. Initiating a study should be such as to maximize its contribution to rational policy making and not to merely slow down the diffusion of a technology. Recently, the Dutch government, advised by the HIEB, has chosen for an (optional) moratorium for new technologies for the time, which is needed to perform a cost-effectiveness study in the context of the 'investigative medicine' programme.

- a shift can be observed from studies dealing with the cost-effectiveness of a specific intervention per se towards studies on the cost-effective use of such intervention in specific patient groups. The latter is sometimes referred to as 'studies on appropriate care', but we would object to this term if it would be defined as 'what doctors seem appropriate'. Indeed, consensus should be reached among all parties in health care about what is appropriate and what not, and economic appraisal is a powerful tool to assist in this debate.

Acknowledgement-We thank Professor M. Drummond for his comments on an earlier draft.

\section{REFERENCES}

1. Hurst J. W. Reforming health care in seven European nations. Hlth Affairs 10, 7-21, 1991.

2. Henry D. Economic analysis as an aid to subsidisation decisions; the development of Australian guidelines for pharmaceuticals. PharmacoEconomics 1, 54-67, 1992.

3. Rutten F. and Haan G. Cost-effective use of medical technology; regulatory instruments and economic incentives. In Policy Making in Ilealth Care; Changing Goals and New Tools (Edited by Jönsson B., Rutten F. and Vang J.). Linköping Collaborating Center, 1990.

4. Offerhaus L. Pillen zonder grenzen? Nederlands Tijd schrift voor Geneeskunde 134, 821-827, 1992.

5. Van de Ven W. P. M. M. From regulated cartel to regulated competition in the Dutch health care system. Eur. Econ. Rev. 34, 632-645, 1990.

6. Henshall C. and Drummond M. Economic appraisal in the British National Health Service: implications of recent developments. Soc. Sci. Med. 38, 1615-1623, 1994.

7. Rutten F. F. H. and Bonsel G. J. High cost technology in health care: a benefit or a burden? Soc. Sci. Med. 35, $567-577,1992$. 